\title{
MODELO BÁSICO PARA EXPLICAR EL PROCESO DE LA TOMOGRAFÍA COMPUTARIZADA USANDO LA RECONSTRUCCIÓN ALGEBRÁICA
}

\author{
Edwin Chávez Ramirez ${ }^{1}$, Efraín Carbajal ${ }^{2}$, Victoriano Yauri Luque ${ }^{3}$, \\ Andrés Guardia Cayo ${ }^{4}$, Cristian Loli Prudencio ${ }^{5}$
}

\begin{abstract}
Resumen: La tomografía computarizada (TC) es uno de los grandes avances tecnológicos utilizados en medicina para el diagnóstico de enfermedades, sin embargo es también una técnica usada en otras áreas, tales como química aeroespacial, radioastronomia, etc. El objetivo de la TC es reconstruir la parte interna de un objeto sin tener la necesidad de abrirlo, dicho procedimiento es conocido como ensayo no destructivo. Para lograr dicho propósito, la TC usa la atenución que sufren los rayos $\mathrm{X}$ al atravesar por dicho objeto con dicha información se reconstruye la parte interna del objeto, es decir un problema inverso. En este artículo nos salimos de la teoría y nos centramos en la práctica mostrando de manera concreta cómo se reconstruye la parte interna de un objeto usando un método algebráico conocido como reconstrucción algebráica.
\end{abstract}

Palabras clave: Tomografía computarizada, reconstrucción algebráica, TC, problema inverso.

\section{BASIC MODEL TO EXPLAIN THE COMPUTED TOMOGRAPHY USING ALGEBRAIC RECONSTRUCTION PROCESS}

\begin{abstract}
Computed tomography (CT) is one of the major technological advances used in medicine to diagnose diseases, but it is also a technique used in other areas, such as eroespacial chemistry, radio astronomy, etc.. The goal of CT is to reconstruct the inside of an object without the need to open it, this process is known a nondestructive testing. To achieve this purpose, the TC uses suffering atenución X-rays to go through said object with said internal information of the object is reconstructed, this is an inverse problem. We got out of the theory and we focus on the practice showing concretely how the inside of an object using an algebraic method known as algebraic reconstruction for a specific event is reconstructed.
\end{abstract}

Keywords:Computed tomography, algebraic reconstruction, CT, inverse problem.

\section{Introducción}

Desde la década de los 70 se han desarrollado diversos métodos (ver [8]) para poder partir a la gente de forma virtual, lo cual es de gran utilidad en la práctica médica. En este artículo, veremos un método sencillo llamado de reconstrucción algebraica (se trata a detalle en [3]). La TC emplea la atenuación que sufren los rayos $X$ al atravesar los tejidos, lo que nos lleva a introducir un pequeño modelo, el cual es una versión simplificada de la llamada ecuación del transporte los detalles se pueden ver en [10].

\footnotetext{
${ }^{1}$ UNMSM, Facultad de Ciencias Matemáticas, e-mail: edwin.chavez@unmsm.edu.pe

${ }^{2}$ UNMSM, Facultad de Ciencias Matemáticas, e-mail: ecarpe@yahoo.com

${ }^{3}$ UNMSM, Facultad de Ciencias Matemáticas, e-mail: victoriano_yauri@hotmail.com

${ }^{4}$ UNMSM, Facultad de Ciencias Matemáticas, e-mail: agcbayo@yahoo.es

${ }^{5}$ UNMSM, Facultad de Ciencias Matemáticas, e-mail: cristianhz@hotmail.com
} 
Es claro que cuando una muestra es atravesada por un fino haz de rayos $X$ de intensidad $I$, la disminución de dicha intensidad depende de la densidad $\rho$ de la muestra ${ }^{1}$ y de su grosor ${ }^{2}$. Si dividimos la zona atravesada por los rayos en pequeñas rodajas transversales de tamaño infinitesimal (ver figura 1), en las que la densidad sea prácticamente constante, es natural suponer que la proporción en que disminuye la intensidad es directamente proporcional a ambas cantidades, digamos con constante de proporcionalidad uno mediante una elección adecuada de las unidades.

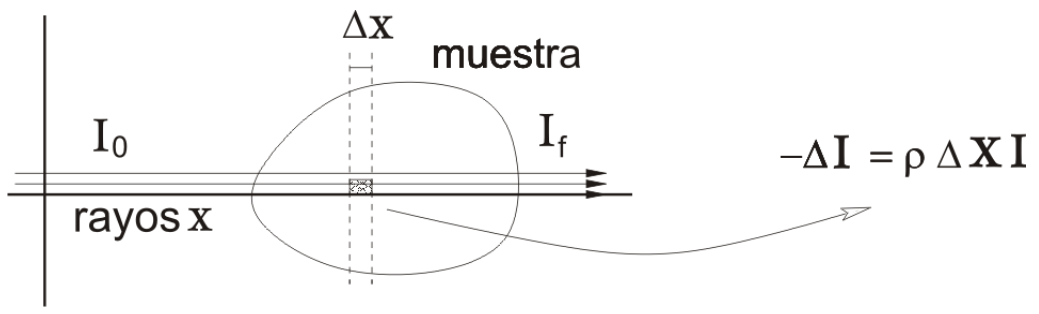

Figura 1: Muestra siendo atravesada por los rayos

Pasando al límite en la anchura de las rodajas, la expresión $-\Delta I=\rho \Delta X I$, se transforma en

$$
-d I=\rho I d x \Rightarrow I^{\prime}=-\rho I \Rightarrow-(\ln I)^{\prime}=\rho .
$$

De modo que si $I_{0}$ es la intensidad inicial (antes de entrar en la muestra) e $I_{f}$ es la intensidad final (después de salir), integrando se tiene

$$
\ln I_{0}-\ln I_{f}=\int \rho .
$$

Evidentemente, si los rayos siguen una recta $L$, en vez del eje $O X$, la integral anterior es la integral de línea a lo largo de $L$.

En definitiva, lo que debemos tener presente es que midiendo intensidades iniciales y finales podemos saber las integrales de la densidad a lo largo de las líneas rectas que siguen los rayos. (Este modelo se conoce como la ley de Beer-Lambert, ver [6]).

Ahora, si la muestra, es una parte del cuerpo humano y hacemos un corte transversal y emitimos un haz delgado de rayos $X$ en dirección de la recta $L$, obtenemos, que nuestra intensidad, varía de acuerdo a la densidad de la muestra, entonces nuestro modelo sería:

$$
\frac{\Delta I}{I}=-f(x, y) \Delta(x, y)
$$

donde $f(x, y)$ es la función que denota la densidad de la muestra en el punto $(x, y)$.

Integrando esta ecuación a lo largo de la recta $L$ obtenemos:

$$
\ln \left(\frac{I_{0}}{I}\right)=\int_{L_{(\delta, s)}} f(x, y) d L,
$$

donde $I_{0}$ es la intensidad del rayo antes de atravesar la muestra e $I$ es la intensidad del rayo después de atravesar la muestra y la recta $L$ tiene la ecuación

$$
L_{(\delta, s)}=\{(s \cos \delta, s \operatorname{sen} \delta)+\mu(-\operatorname{sen} \delta, \cos \delta) ; \mu \in \mathbb{R}, \delta \in(0, \pi]\} .
$$

Ahora podemos calcular esta integral de línea sobre cada una de las rectas.

$$
\mathcal{R} f(s, \delta)=\int_{L_{(\delta, s)}} f(x, y) d L .
$$

\footnotetext{
${ }^{1} \mathrm{Si}$ los rayos $X$ atraviesan plomo es distinto a que atraviesen el aire

${ }^{2} \mathrm{Si}$ atraviesan un muro de dos metros atenúa menos que otro de cinco
} 
El operador que asigna a una función escalar su integral de línea sobre cada recta es lo que se llama la Transformada de Radon de $f$. (Ver la sección 1.2 de [3])

El problema de calcular la transformada de Radon $(\mathcal{R} f)$ cuando se conoce el valor de $f$, es conocido como problema directo, mientras que determinar la densidad $f$ cuando se conoce la transformada de Radon $\mathcal{R} f$ es conocido como problema inverso, más detalles se pueden ver en [1], [3], [5], [7].

Matemáticamente podemos decir que la reconstrucción tomográfica trata de mostrar una imagen a partir de sus proyecciones ${ }^{3}$, y la solución matemática fue descubierta en 1917 por el matemático Johann Radon. El problema es hallar la fórmula para la inversa de la transformada de Radon. La dificuldad con la fórmula de inversión de Radon es que no nos proporciona un algoritmo que pueda ser implementado computacionalmente, de allí la necesidad de buscar otros métodos ${ }^{4}$ los cuales muestren un algoritmo que pueda ser implementado computacionalmente.

\section{Preliminares}

\section{Teorema 2.1}

Sea un sistema compatible determinado por $N$ ecuaciones con $N$ incognitas:

$$
\left(\overrightarrow{f_{1}}, \vec{x}\right)=b_{1}, \quad\left(\overrightarrow{f_{2}}, \vec{x}\right)=b_{2}, \quad\left(\vec{f}_{3}, \vec{x}\right)=b_{3}, \ldots\left(\vec{f}_{N}, \vec{x}\right)=b_{N},
$$

donde $\vec{x}=\left(x_{1}, x_{2}, \ldots x_{N}\right)$ es el vector de incógnitas. Introduciendo las aplicaciones afines $L_{i}: \mathbb{R}^{N} \rightarrow \mathbb{R}^{N}$ definidas como

$$
L_{i}(\vec{x})=P_{i}(x)+b_{i} \frac{\vec{f}_{i}}{\left\|\vec{f}_{i}\right\|^{2}} \quad \text { con } \quad P_{i}(\vec{x})=\vec{x}-\left(\overrightarrow{f_{i}}, \vec{x}\right) \frac{\vec{f}_{i}}{\left\|\vec{f}_{i}\right\|^{2}}
$$

se tiene que para cualquier $\vec{x}_{0} \in \mathbb{R}^{N}$, el algoritmo iterativo

$$
\vec{x}_{n+1}=\left(L_{N} \circ L_{N-1} \circ \ldots \circ L_{2} \circ L_{1}\right)\left(\vec{x}_{n}\right)
$$

genera una sucesión que converge a la solución del sistema.

\section{Demostración.}

Primeramente veamos que $\vec{P}_{i}(\vec{v})$ es el operador proyección ([4]). En efecto:

- Probemos que $P_{i}$ es una proyección, i.e., debemos probar que

$$
P_{i}\left(P_{i}(\vec{v})\right)=P_{i}(\vec{v}) \text {, para todo } \vec{v} \in \mathbb{R}^{N}
$$

$$
\begin{aligned}
P_{i}\left(P_{i}(\vec{v})\right): & =P_{i}(\vec{v})-\left(\vec{f}_{i}, P_{i}(\vec{v})\right) \frac{\vec{f}_{i}}{\left\|\vec{f}_{i}\right\|^{2}} \\
& =P_{i}(\vec{v})-\left(\vec{f}_{i}, \vec{v}-\left(\overrightarrow{f_{i}}, \vec{v}\right) \frac{\vec{f}_{i}}{\left\|\vec{f}_{i}\right\|^{2}}\right) \frac{\overrightarrow{f_{i}}}{\left\|\vec{f}_{i}\right\|^{2}} \\
& =P_{i}(\vec{v})-\left(\overrightarrow{f_{i}}, \vec{v}\right) \frac{\overrightarrow{f_{i}}}{\left\|\vec{f}_{i}\right\|^{2}}+\left(\vec{f}_{i}, \vec{v}\right)\left(\vec{f}_{i}, \frac{\overrightarrow{f_{i}}}{\left\|\vec{f}_{i}\right\|^{2}}\right) \frac{\vec{f}_{i}}{\left\|\vec{f}_{i}\right\|^{2}} \\
& =P_{i}(\vec{v})-\left(\overrightarrow{f_{i}}, \vec{v}\right) \frac{\overrightarrow{f_{i}}}{\left\|\vec{f}_{i}\right\|^{2}}+\left(\overrightarrow{f_{i}}, \vec{v}\right) \frac{\vec{f}_{i}}{\left\|\vec{f}_{i}\right\|^{2}} \\
& =P_{i}(\vec{v}) .
\end{aligned}
$$

Por lo tanto $P_{i}$ es una proyección.

\footnotetext{
${ }^{3}$ Básicamente, una proyección en un ángulo dado, es un conjunto de integrales de línea en dicha dirección

${ }^{4}$ que calculen la inversa de la transformada de Radon
} 
- Probemos que $P_{i}(\vec{v})$ proyecta el vector $\vec{v}$ sobre el hiperplano $\left(\vec{f}_{i}, \vec{x}\right)=0$, i.e., debemos $\operatorname{probar}\left(\vec{f}_{i}, P_{i}(\vec{v})\right)=0$, en efecto tenemos:

$$
\begin{aligned}
\left(\overrightarrow{f_{i}}, P_{i}(\vec{v})\right) & =\left(\overrightarrow{f_{i}}, \vec{v}-\left(\vec{f}_{i}, \vec{v}\right) \frac{\vec{f}_{i}}{\left\|\vec{f}_{i}\right\|^{2}}\right) \\
& =\left(\overrightarrow{f_{i}}, \vec{v}\right)-\left(\overrightarrow{f_{i}}, \vec{v}\right)\left(\vec{f}_{i}, \frac{\vec{f}_{i}}{\left\|\vec{f}_{i}\right\|^{2}}\right) \\
& =0 .
\end{aligned}
$$

Por lo tanto $P_{i}(\vec{v})$ está en el hiperplano $\left(\vec{f}_{i}, \vec{x}\right)=0$.

- Probemos que $L_{i}$ es también un operador proyección, i.e., debemos probar que $L_{i}\left(L_{i}(\vec{v})\right)=$ $L_{i}(\vec{v})$, para todo $\vec{v} \in \mathbb{R}^{N}$

$$
\begin{aligned}
& L_{i}\left(L_{i}(\vec{v})\right):=P_{i}\left(L_{i}(\vec{v})\right)+b_{i} \frac{\overrightarrow{f_{i}}}{\left\|\vec{f}_{i}\right\|^{2}} \\
& =L_{i}(\vec{v})-\left(\vec{f}_{i}, L_{i}(\vec{v})\right) \frac{\vec{f}_{i}}{\left\|\vec{f}_{i}\right\|^{2}}+b_{i} \frac{\vec{f}_{i}}{\left\|\vec{f}_{i}\right\|^{2}} \\
& =L_{i}(\vec{v})-\left(\vec{f}_{i}, P_{i}(\vec{v})+b_{i} \frac{\overrightarrow{f_{i}}}{\left\|\vec{f}_{i}\right\|^{2}}\right) \frac{\overrightarrow{f_{i}}}{\left\|\vec{f}_{i}\right\|^{2}}+b_{i} \frac{\vec{f}_{i}}{\left\|\vec{f}_{i}\right\|^{2}} \\
& =L_{i}(\vec{v})-\left(\vec{f}_{i}, P_{i}(\vec{v})\right) \frac{\vec{f}_{i}}{\left\|\vec{f}_{i}\right\|^{2}}-b_{i}\left(\vec{f}_{i}, \frac{\vec{f}_{i}}{\left\|\vec{f}_{i}\right\|^{2}}\right) \frac{\vec{f}_{i}}{\left\|\vec{f}_{i}\right\|^{2}}+b_{i} \frac{\vec{f}_{i}}{\left\|\vec{f}_{i}\right\|^{2}} \\
& =L_{i}(\vec{v})-\left(\vec{f}_{i}, P_{i}(\vec{v})\right) \frac{\vec{f}_{i}}{\left\|\vec{f}_{i}\right\|^{2}} \\
& =L_{i}(\vec{v})-\left(\overrightarrow{f_{i}}, \vec{v}-\left(\overrightarrow{f_{i}}, \vec{v}\right) \frac{\overrightarrow{f_{i}}}{\left\|\vec{f}_{i}\right\|^{2}}\right) \frac{\vec{f}_{i}}{\left\|\vec{f}_{i}\right\|^{2}} \\
& =L_{i}(\vec{v})-\left(\overrightarrow{f_{i}}, \vec{v}\right) \frac{\vec{f}_{i}}{\left\|\vec{f}_{i}\right\|^{2}}+\left(\overrightarrow{f_{i}}, \vec{v}\right)\left(\vec{f}_{i}, \frac{\vec{f}_{i}}{\left\|\vec{f}_{i}\right\|^{2}}\right) \frac{\vec{f}_{i}}{\left\|\vec{f}_{i}\right\|^{2}} \\
& =L_{i}(\vec{v})-\left(\overrightarrow{f_{i}}, \vec{v}\right) \frac{\overrightarrow{f_{i}}}{\left\|\vec{f}_{i}\right\|^{2}}+\left(\overrightarrow{f_{i}}, \vec{v}\right) \frac{\overrightarrow{f_{i}}}{\left\|\vec{f}_{i}\right\|^{2}} \\
& =L_{i}(\vec{v}) .
\end{aligned}
$$

Por lo tanto $L_{i}$ es un operador proyección. 
- Veamos que $L_{i}(\vec{v})$ proyecta el vector $\vec{v}$ sobre el hiperplano $\left(\vec{f}_{i}, \vec{x}\right)=b_{i}$, i.e., $\left(\vec{f}_{i}, L_{i}(\vec{v})\right)=b_{i}$, en efecto tenemos:

$$
\begin{aligned}
\left(\overrightarrow{f_{i}}, L_{i}(\vec{v})\right) & =\left(\vec{f}_{i}, P_{i}(\vec{v})+b_{i} \frac{\vec{f}_{i}}{\left\|\vec{f}_{i}\right\|^{2}}\right) \\
& =\left(\vec{f}_{i}, P_{i}(\vec{v})\right)+b_{i}\left(\vec{f}_{i}, \frac{\vec{f}_{i}}{\left\|\vec{f}_{i}\right\|^{2}}\right) \\
& =\left(\overrightarrow{f_{i}}, P_{i}(\vec{v})\right)+b_{i} \\
& =\left(\overrightarrow{f_{i}}, \vec{v}-\left(\overrightarrow{f_{i}}, \vec{v}\right) \frac{\vec{f}_{i}}{\left\|\vec{f}_{i}\right\|^{2}}\right)+b_{i} \\
& =\left(\overrightarrow{f_{i}}, \vec{v}\right)-\left(\overrightarrow{f_{i}}, \vec{v}\right)\left(\vec{f}_{i}, \frac{\vec{f}_{i}}{\left\|\vec{f}_{i}\right\|^{2}}\right)+b_{i} \\
& =b_{i} .
\end{aligned}
$$

Por lo tanto $L_{i}(\vec{v})$ proyecta el vector $\vec{v}$ sobre el hiperplano $\left(\overrightarrow{f_{i}}, \vec{x}\right)=b_{i}$.

Observación 2.2 Nótese que $P_{i}(\vec{x})$ es la proyección cuando el hiperplano correspondiente $L_{i}(\vec{x})$ se traslada al origen.

Por otro lado, para cualquier $\vec{v} \in \mathbb{R}^{N}$ se tiene que $\left\|P_{1}(\vec{v})\right\|<\|\vec{v}\|$, excepto si $\vec{v}$ pertenece al plano, en cuyo caso tendríamos $\left(\vec{f}_{i}, \vec{v}\right)=0$ y también $P_{1}(\vec{v})=\vec{v}$. De la misma forma $\left\|\left(P_{2} \circ P_{1}\right)(\vec{v})\right\|<\|\vec{v}\|$, excepto si $\left(\vec{f}_{1}, \vec{v}\right)=\left(\overrightarrow{f_{2}}, \vec{v}\right)=0$, si seguimos este mismo argumento sucesivamente hasta llegar a $N$, donde se tendría $\left\|\left(P_{N} \circ P_{N-1} \circ \ldots \circ P_{2} \circ P_{1}\right)(\vec{v})\right\|<\|\vec{v}\|$, excepto si

$$
\left(\overrightarrow{f_{1}}, \vec{v}\right)=\left(\overrightarrow{f_{2}}, \vec{v}\right)=\left(\overrightarrow{f_{3}}, \vec{v}\right)=\ldots=\left(\vec{f}_{N-1}, \vec{v}\right)=\left(\vec{f}_{N}, \vec{v}\right)=0,
$$

como por hipótesis tenemos que el sistema es compatible, es decir que las $\vec{f}_{i}$ son linealmente independientes, entonces la única solución de

$$
\left(\vec{f}_{1}, \vec{v}\right)=\left(\vec{f}_{2}, \vec{v}\right)=\left(\vec{f}_{3}, \vec{v}\right)=\ldots=\left(\vec{f}_{N-1}, \vec{v}\right)=\left(\vec{f}_{N}, \vec{v}\right)=0
$$

es la trivial.

Luego, podemos concluir que $\left\|\left(P_{N} \circ P_{N-1} \circ \ldots \circ P_{2} \circ P_{1}\right)(\vec{v})\right\| \leq C\|\vec{v}\|$ para algún $C<1$, podemos también decir que $\left\|P_{N} \circ P_{N-1} \circ \ldots \circ P_{2} \circ P_{1}\right\| \leq C<1$.

Observación 2.3 Notar que por la compacidad de la bola unitaria se tiene que el operador

$$
\left\|\left(P_{N} \circ P_{N-1} \circ \ldots \circ P_{2} \circ P_{1}\right)(\vec{v} /\|\vec{v}\|)\right\|
$$

alcanza un máximo, menor que 1 , en $\mathbb{R}^{N}-\{\overrightarrow{0}\}$.

Consideremos el operador $Q: \mathbb{R}^{N} \rightarrow \mathbb{R}^{N}$ definido como

$$
Q=L_{N} \circ L_{N-1} \circ \ldots \circ L_{2} \circ L_{1},
$$

Veamos que $Q$ así definido es un operador de contracción. En efecto tenemos

$$
\begin{aligned}
\|Q(\vec{x})-Q(\vec{y})\| & =\left\|\left(L_{N} \circ \ldots \circ L_{1}\right)(\vec{x})-\left(L_{N} \circ \ldots \circ L_{1}\right)(\vec{y})\right\| \\
& =\left\|\left(L_{N} \circ L_{N-1} \circ \ldots \circ L_{2} \circ L_{1}\right)(\vec{x}-\vec{y})\right\| \\
& =\left\|\left(P_{N} \circ P_{N-1} \circ \ldots \circ P_{2} \circ P_{1}\right)(\vec{x}-\vec{y})\right\| \\
& \leq C\|x-y\|
\end{aligned}
$$


Como $C<1$ podemos concluir que $Q$ es un operador de contracción.

Por otro lado por el teorema del punto fijo (ver teorema asegura que tiene un solo punto fijo que puede obtenerse con el algoritmo iterativo

$$
\vec{x}_{n+1}=\left(L_{N} \circ L_{N-1} \circ \ldots \circ L_{2} \circ L_{1}\right)\left(\overrightarrow{x_{n}}\right)
$$

para cualquier $x_{0} \in \mathbb{R}^{N}$. Este punto fijo es la solución del sistema lineal (3), ya que los $L_{i}$ dejan invariante a dicha solución pues al estar dicha solución en todos los hiperplanos es invariante bajo proyecciones sobre ellos.

Una ventaja del método es que se puede aplicar incluso en el caso en el que exista más ecuaciones que incógnitas simplemente aumentando el valor de $N$ en el sistema (3). Así pues haciendo más mediciones de las que necesitamos podemos estar bastante seguros de que el sistema no es indeterminado, aunque corremos el riesgo que por errores experimentales o en el modelo, el sistema sea incompatible. Incluso en este caso, si los errores no son muy graves, la solución $\vec{x}_{n}$ oscilará alrededor de la solución real.

\section{Metodología}

Los algoritmos de reconstrucción algebraica comienzan considerando una versión discretizada de la sección que se quiere examinar. Con tal fin, introducimos una malla cuadrada de $M \times M$ cuadraditos. Si la malla es suficientemente fina, la densidad es aproximadamente constante en cada cuadradito. Así que se puede considerar que hay una matriz de densidades $M \times M$ donde el elemento $\rho_{i j}$ es la densidad en el cuadradito $c_{i j}$. Por otra parte, la atenuación de un rayo a lo largo de una recta $L$ permite conocer $\int_{L} f(x, y) d L$, que en esta versión digitalizada, se aproxima por una suma de Riemann, y de hecho coincide con ella suponiendo $f(x, y)$ es realmente constante en cada $c_{j}$.

$$
\ln \left(\frac{I_{0}}{I}\right)=\int_{L} f(x, y) d L=\sum f_{j}\left|c_{i} \cap L\right|
$$

Aparentemente el problema ya está resuelto: queremos calcular el valor de las incógnitas $x_{1}=\rho_{11}, x_{2}=\rho_{12}, x_{3}=\rho_{13}, \ldots x_{M^{2}}=\rho_{M M}$ y, según (5), para cada rayo tenemos una ecuación lineal en estas incógnitas; basta tomar un número suficiente de rayos y resolver el sistema lineal correspondiente.

Puede que esto resuelva el problema desde el punto de vista teórico, pero la aplicación práctica requiere ir más allá. Supongamos por ejemplo que deseamos tener una resolución comparable a la de un monitor y para ello imaginamos una malla de $1000 \times 1000$ pixels que contiene la sección del cuerpo humano que vamos a examinar (en [6]) se apunta $256 \times 256$ pixels como una resolución posible en la práctica, y por la imágenes allí mostradas $128 \times 128$ pudiera ser a veces deficiente). Entonces habrá 106 incógnitas $\rho_{i j}$ que calcular.

El sistema lineal correspondiente tendrá una matriz de $106 \times 106=1012$ elementos lo cual podrá causar algunos problemas de memoria en ordenadores convencionales si los tenemos que almacenar todos (necesitaríamos algo comparable a un Terabyte de memoria libre). Las estimaciones generales del número de operaciones para resolver un sistema lineal por eliminación de Gauss es del orden del cubo del número de variables, en nuestro caso 1018. A una velocidad de $1 G H z$ esto llevaría del orden de 30 años.

Necesitamos, por tanto, un método maravilloso que requiera incomparablemente menos operaciones que el de Gauss. Quizá tal método no exista en general, pero aquí estamos considerando sistemas muy especiales y hay esperanzas sobre todo si nos contentamos con soluciones aproximadas. Nótese que típicamente un rayo atraviesa $M$ cuadraditos (pixels), con lo cual en cada ecuación sólo aparecen $M$ incógnitas de las $M^{2}$ que hay en total. Es decir, la matriz de coeficientes es muy dispersa, está llena de ceros.

Vamos a mostrar un método iterativo creado por S. Kaczmarz en 1937 que no altera la dispersión de la matriz, de hecho no modifica la matriz de coeficientes, lo que redunda en que las operaciones sólo se hacen con los "pocos" coeficientes no nulos. La idea subyacente es la generalización a 
dimensiones mayores de un hecho muy sencillo: Podemos aproximar el punto donde se cortan dos rectas en $R^{2}$ partiendo de un punto cualquiera y proyectando alternativamente en cada una de las rectas, leuego entonces si usamos el teorema 2.1, podemos resolver el problema.

\section{Experimento}

El objetivo es explicar las nociones básicas de uno de los métodos con los que se hacen tomografías y su relación con los algoritmos iterativos para resolver sistemas lineales, para ello usaremos los siguientes materiales:

- Cartulina.

- Plástico semitransparente (por ejemplo papel celofán).

- Una linterna.

- Una calculadora.

Como todos sabemos los tejidos del cuerpo humano no se traslucen, entonces no podemos cambiar en las aplicaciones médicas los rayos $X$ por la luz visible; lo que vamos a hacer aquí es sustituir los tejidos por unos cubitos translúcidos con los que podamos ilustrar como se reconstruye la parte interna de un objeto usando el método de reconstrucción algebraica.

Con la cartulina fabricaremos nueve cubos y en sus caras laterales abriremos "ventanas" para que pueda pasar la luz, las cuales cubriremos algunos de ellos con el plástico semitransparente. $\mathrm{Al}$ poner tres cubos seguidos y enfocarlos con la luz de la linterna, se pueden detectar en una pantalla (una hoja de papel) cuatro posibles intensidades dependiendo de si ninguno, uno, dos o los tres cubos tienen plástico en sus ventanas, como se ilustra en la figura 2 .

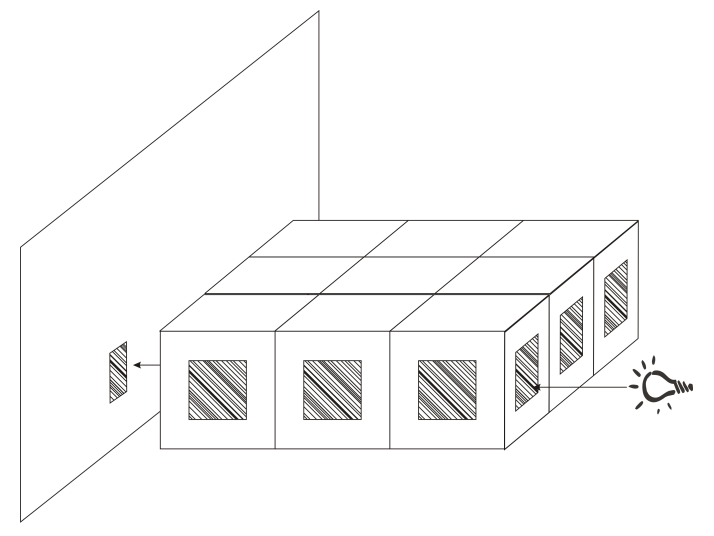

Figura 2: Los cubos siendo atravesados por la luz.

Convencionalmente designaremos estas intensidades por $I=1 ; 1 / 2 ; 1 / 3 ; 1 / 4$ respectivamente. Para llevar a cabo el experimento, es importante familiarizarse con las intensidades de manera que podamos distinguirlas a simple vista. Si esto no es posible, debemos cambiar el tipo de plástico.

Dispongamos los cubos formando un cuadrado, como se muestra en la figura 3 , donde los cuadraditos pintados significan que son cubitos que tienen sus ventanas cubiertas con plastico.

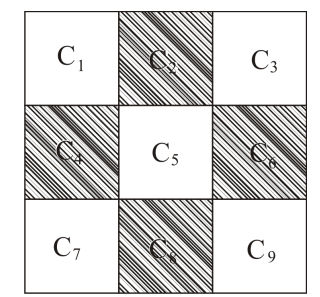

Figura 3: Formando un cuadrado con los cubos. 
Usando la ley de Beer-Lambert, tenemos

$$
\frac{\Delta I}{I}=-f(x) \Delta x
$$

Podemos reescribirla como

$$
-\frac{I_{f}-I_{i}}{I_{f}}=f(x) \Delta x
$$

Donde:

* $I_{f}$ : Es la intensidad final (al momento de la salida).

* $I_{i}$ : Es la intensidad inicial (al momento de la entrada).

* $f(x)$ : Es el coeficiente de densidad (lo que estamos por reconstruir).

* $\Delta x$ : Es la intersección del rayo con el cubo (en este caso será igual a 1).

Denotemos por $C_{j}$ el coeficiente de densidad del $j$-ésimo cuadradito, entonces $f(x)$ puede ser reescrito como una sumatoria, además estamos asumiendo que $I_{i}=1$, luego la ecuación (6), se transforma en

$$
I_{f}^{-1}-1=\sum_{j=1}^{3} C_{j}
$$

Si hacemos pasar la luz de la linterna en cada una de las direcciones (horizontales, verticales y diagonales) como se muestra en la figura 4.

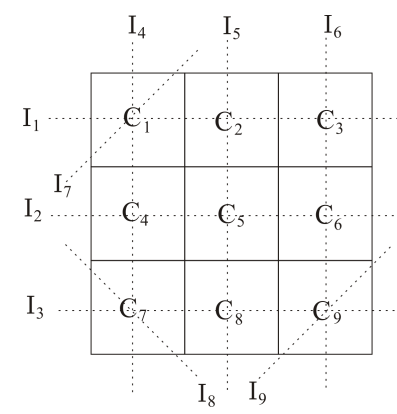

Figura 4: Forma en que los rayos de luz atraviesan el cuerpo.

Entonces luego obtenemos las siguientes ecuaciones

$$
\begin{aligned}
& C_{1}+C_{2}+C_{3}=I_{f_{1}}^{-1}-1 \\
& C_{4}+C_{5}+C_{6}=I_{f_{2}}^{-1}-1 \\
& C_{7}+C_{8}+C_{9}=I_{f_{3}}^{-1}-1 \\
& C_{1}+C_{4}+C_{7}=I_{f_{4}}^{-1}-1 \\
& C_{2}+C_{5}+C_{8}=I_{f_{5}}^{-1}-1 \\
& C_{3}+C_{6}+C_{9}=I_{f_{6}}^{-1}-1 \\
& C_{1}=I_{f_{7}}^{-1}-1 \\
& C_{7}=I_{f_{8}}^{-1}-1 \\
& C_{9}=I_{f_{9}}^{-1}-1
\end{aligned}
$$

Obteniendo así un conjunto de 9 ecuaciones con 9 incógnitas, supongamos que numeramos los cubos como en la figura 4 y asignamos al cubo $j$-ésimo el valor $C_{j}=0$, si está hueco y $C_{j}=1$, si es semitransparente. Luego, para esta ordenación (de los cubitos), obtenemos las siguientes intensidades finales

$$
\vec{I}=\left(I_{1}, I_{2}, \ldots, I_{8}, I_{9}\right)=\left(\frac{1}{2}, \frac{1}{3}, \frac{1}{2}, \frac{1}{2}, \frac{1}{3}, \frac{1}{2}, 1,1,1\right) .
$$


Luego, reemplazando estas intensidades en las ecuaciones de (7), obtenemos

$$
\begin{aligned}
& C_{1}+C_{2}+C_{3}=1 \\
& C_{4}+C_{5}+C_{6}=2 \\
& C_{7}+C_{8}+C_{9}=1 \\
& C_{1}+C_{4}+C_{7}=1 \\
& C_{2}+C_{5}+C_{8}=2 \\
& C_{3}+C_{6}+C_{9}=1 \\
& C_{1}=0 \\
& C_{7}=0 \\
& C_{9}=0
\end{aligned}
$$

Podemos verificar que el sistema (8) es un sistema incompatible indeterminado, ya que si ponemos al sistema matricialmente, este tiene determinante 0. Entonces para conseguir un sistema determinado, añadimos una ecuación más que es la que pasa por la esquina de $C_{3}$, entonces tendría una intensidad $I_{10}=1$.

Evidentemente si en vez de nueve celdillas tuviéramos miles, sería muy costoso de comprobar si el sistema es determinado, y en la práctica simplemente añadiríamos más ecuaciones de las necesarias, pensando que habría que tener muy mala suerte para que todavía el rango de la matriz no fuera el adecuado.

El sistema se resuelve directamente haciendo los cálculos respectivos, y la solución es $\vec{C}=$ $\left(C_{1}, \ldots C_{9}\right)=(0,1,0,1,0,1,0,1,0)$. Pero como queremos ilustrar la reconstrucción algebraica, usaremos el algoritmo que se muestra en el teorema 2.1

$$
\vec{x}_{n+1}=\left(L_{10} \circ L_{9} \circ \ldots \circ L_{2} \circ L_{1}\right)\left(\vec{x}_{n}\right)
$$

partiendo de $\vec{x}=\overrightarrow{0}$. Donde $L_{i}$ son las proyecciones en los hiperplanos que definen las ecuaciones de (8) y la añadida después (de hecho podríamos reemplazar una de las ecuaciones por ella). Con una calculadora y un poco de paciencia, se pueden hacer una o dos iteraciones. Usando el programa $(4.1)^{5}$ de la siguiente sección, se puede calcular con mucha más facilidad las iteraciones como por ejemplo, algunos de los $\vec{x}_{n}$ obtenidos de esta forma son

$$
\begin{aligned}
& \vec{x}_{5}=(0,0.7225,0,0.7225,0.5549,0.7225,0,0.7225,0) \\
& \vec{x}_{10}=(0,0.8460,0,0.8460,0.3079,0.8460,0,0.8460,0) \\
& \vec{x}_{15}=(0,0.9146,0,0.9146,0.1709,0.9146,0,0.9146,0) \\
& \vec{x}_{25}=(0,0.9737,0,0.9737,0.0526,0.9737,0,0.9737,0) \\
& \vec{x}_{50}=(0,0.9986,0,0.9986,0.0028,0.9986,0,0.9986,0)
\end{aligned}
$$

Nótese que la aproximación de $\vec{x}_{n}$ a la solución hace posible adivinar enseguida dónde están los cubos semitransparentes $\left(C_{i}=1\right)$ y los huecos $\left(C_{i}=0\right)$. En el límite $\vec{x}_{n} \rightarrow \vec{C}$.

Si adicionalmente nos apetece mostrar una vista de la ubicación de los cubitos, con el programa (4.1), obtenemos la figura 5, si bien es cierto esta gráfica no nos da más información, pero se ve bonita.

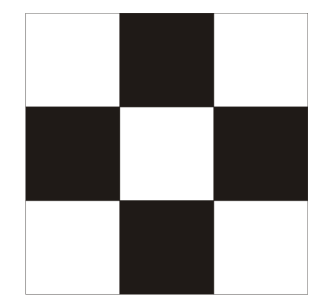

Figura 5: Grafica.

\footnotetext{
${ }^{5}$ Este programa se usa con $I_{f}=\left(I_{1}, I_{2}, \ldots, I_{9}, I_{10}\right)=\left(\frac{1}{2}, \frac{1}{3}, \frac{1}{2}, \frac{1}{2}, \frac{1}{3}, \frac{1}{2}, 1,1,1,1\right)$.
} 
Adicionalmente hacemos más experimentos, teniendo presente el orden de los rayos como se muestra en la figura 6 .

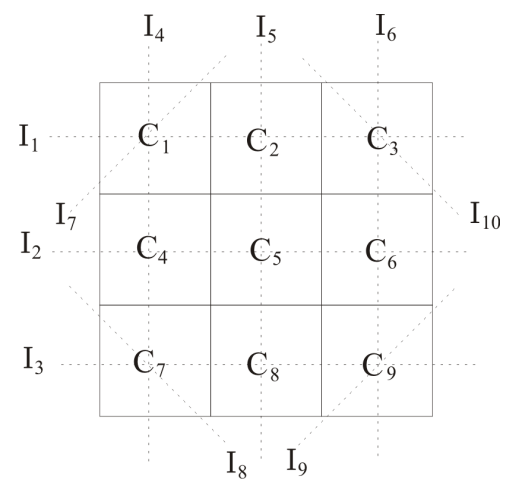

Figura 6: Orden en que son tomados los rayos.

Seguimos tomando $\vec{x}=\overrightarrow{0}$, y consideramos las distribuciones de los cubitos:

- Si usamos la distribución que se muestra en la figura 7

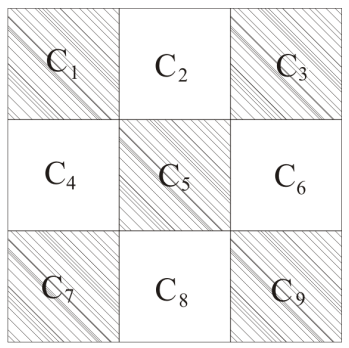

Figura 7: Distribución de los cubitos

Entonces las intensidades finales serán

$$
I_{f}=(1 / 3,1 / 2,1 / 3,1 / 3,1 / 2,1 / 3,1 / 2,1 / 2,1 / 2,1 / 2)
$$

luego usando el programa (4.1) de la siguiente sección obtenemos

$$
\vec{x}_{50}=(1,0.0263,1,0.0263,0.9474,0.0263,1,0.0263,1)
$$

Usando el programa (4.2) de la siguiente sección obtenemos la figura 8

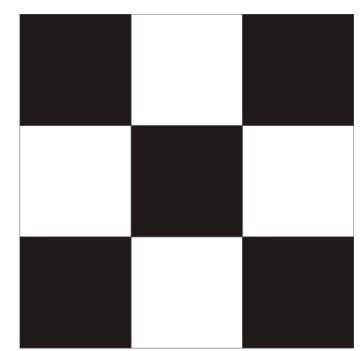

Figura 8: Gráfica. 


\section{Programas para la reconstrucción algebraica}

\section{Programa 4.1}

Tipo:Matlab

Descripción: Hace las iteraciones para mostrar la reconstrucción algebraica, se guardó con el nombre tomografía.m, se resalta el nombre pues el programa (4.2) hace uso de este programa, los datos de entrada son $\vec{x}_{0}$ y el número de iteraciones $n$, arroja como respuesta la iteración $\vec{x}_{n}$, para correrlo ingresamos $x=$ zeros $(9,1)$ y el $n$ deseado.

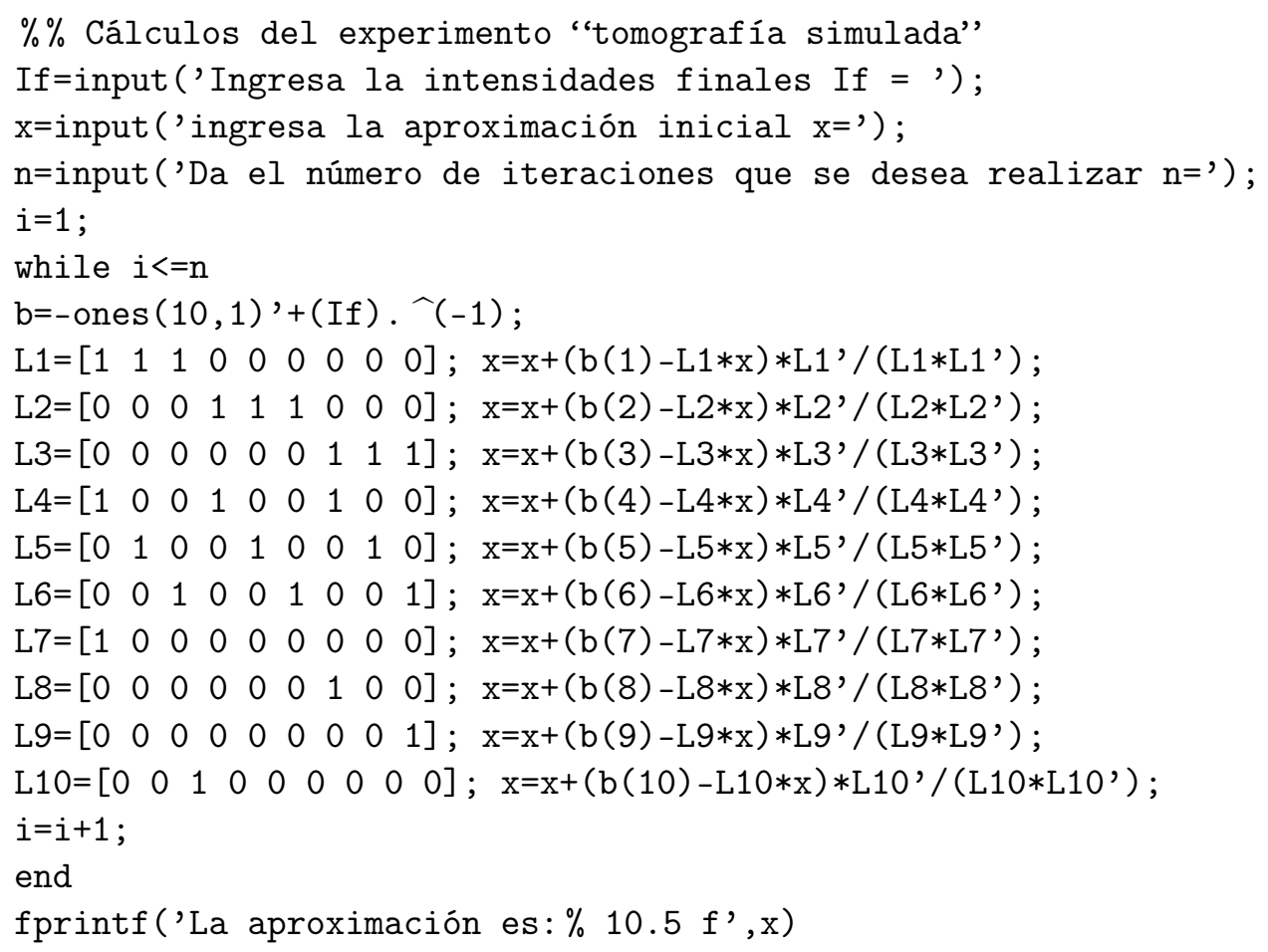

\section{Programa 4.2}

Tipo:Matlab

Descripción: Hace las gráficas de la forma en que están distribuidos los cubos, usando la reconstrucción algebraica, usa el programa anterior.

$\% \% \% \% \% \% \% \%$ INICIO $\% \% \% \% \% \% \% \%$

$\% \%$ RECONSTRUCCIÓN ALGEBRAICA DE UNA MUESTRA

$I=$ ones $(256,256)$;

$\% \%$ VALORES INICIALES

$\% \% \mathrm{n}=0$

$\% \% \mathrm{x}=$ zeros $(9,1)$

$\% \%$ LLAMADA AL PROGRAMA ANTERIOR

tomografia

$\mathrm{n}=\mathrm{n}+1$

$\% \% \% \% \% \%$ DIBUJA $\% \% \% \% \% \% \%$

Imag $=[\mathrm{x}(1) * \mathrm{I} \times \mathrm{x}(2) * \mathrm{I} \mathrm{x}(3) * \mathrm{I} ; \ldots$

$\mathrm{x}(4) * \mathrm{I} \quad \mathrm{x}(5) * \mathrm{I} \quad \mathrm{x}(6) * \mathrm{I} ; \ldots$

$\mathrm{x}(7) * \mathrm{I} \quad \mathrm{x}(8) * \mathrm{I} \quad \mathrm{x}(9) * \mathrm{I}]$;

imshow (1-Imag) 


\section{Conclusiones}

- Es posible reconstruir la estructura interna de los objetos, sin necesidad de abrirlos.

- Existen otras fórmulas de reconstrucción $y=\mathcal{K} x$

- Una variante sería tomar proyecciones en otra forma como por ejemplo en forma de abanico (ver figura 9):

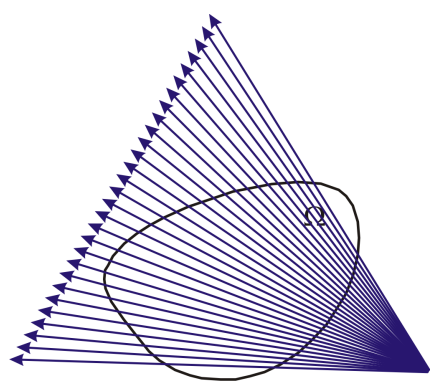

Figura 9: Proyección en forma de abanico. 


\section{REFERENCIAS BIBLIOGRÁFICAS}

[1] Carbajal Peña E. (1999) Problemas inversos y sus aplicaciones. Serie A:Monografías, Colección de Matemáticos del Perú

[2] Chamizo Fernando. (2003) Notas del curso Modelización II.

[3] Chávez Ramírez E. (2009) Solución al Problema Inverso Tomografía Computarizada Tratamiento Numérico. Tesis de Licenciatura.

[4] C.W. Groetsch. (1999) Inverse Problems: activites for undergraduates. Mathematical Association of America.

[5] Hansen Chistian Per. (1998) Rank-Deficient and Discrete Ill-posed Problems. Technical University of Denmark. Siam.

[6] Kak, A.C. Slaney, M. (1998) Principles of Computerized Tomographic Imaging. New York: I. Press. Versión electrónica Iam.

[7] Kirsch Andreas. (1996) An Introduction to the Mathematical Theory of Inverse Problems. Texts in Applied Mathematics. Springer.

[8] Natterer. F.(2001) The Mathematics of Computerized tomography. (New York: Wiley)

[9] Quarin. Tomografía por rayos X. Acceso: nov. 2007. Disponible en: www.geocities.com/tomografiademadeira/autor2.html.

[10] A.G. Ramm, A.I. Katsevich. The Radon Transform and Local Tomography. CRC Press. 